months when vitamin D levels are lowest. The purpose of this study was to investigate the local and systemic effects of vitamin $\mathrm{D}$ deficiency in a murine model of sepsis-induced lung injury where we can predictably time the initiating insult.

Methods We fed 8 wild-type $\mathrm{C} 57 \mathrm{Bl} / 6$ mice a diet completely devoid of vitamin D for 6 weeks to induce severe vitamin D deficiency $(9 \mathrm{nmol} / \mathrm{l})$ and compared to 7 mice fed a vitamin D sufficient diet $(42 \mathrm{nmol} / \mathrm{l})$. Caecal ligation and puncture (CLP) was used to establish sepsis. Animals were culled 16h after CLP and blood, peritoneal lavage fluid (PLF) and bronchoalveloar lavage fluid (BALF) were collected. Cell infiltrates were assessed by flow cytometry. Fluid protein levels were measured and tissue protein permeability index (PPI) was calculated as the ratio between fluid and serum protein. Bacterial load was evaluated as colony-forming units (CFU) after $24 \mathrm{~h}$ incubation on appropriate media.

Results Vitamin D deficient mice had increased bacterial load in BALF, blood and PLF compared to dietary sufficient mice. BALF protein permeability index was higher in deficient compared to sufficient mice but there was no difference in cell numbers recruited to the lung. PLF protein permeability index was also increased in the deficient group compared to sufficient mice with an associated significant increase in neutrophils recruited to the peritoneum. (See Table 1)

Conclusion Vitamin D deficiency significantly increases the bacterial load both systemically, locally and within the lung in a murine model of peritonitis. This is associated with an increase in tissue permeability locally and within the lung. These data support pre-existing vitamin D deficiency as a determinant of the severity of bacteraemic sepsis and may account for some of the seasonal variations observed in the incidence of sepsis.

Abstract S98 Table 1. Differences between dietary deficient and sufficient mice post CLP induced sepsis. Data is expressed as median values.

\begin{tabular}{|c|c|c|c|}
\hline & $\begin{array}{l}\text { Sufficient } \\
(\mathrm{n}=7)\end{array}$ & $\begin{array}{l}\text { Deficient } \\
(\mathrm{n}=8)\end{array}$ & $p$-value \\
\hline Bacteria (CFU $\left.\times 10^{3}\right)$ & 0.51 & 2.51 & 0.038 \\
\hline BALF & 0.28 & 66.1 & 0.019 \\
\hline Blood & 8.22 & 336.6 & 0.005 \\
\hline \multicolumn{4}{|l|}{ PLF } \\
\hline PPI (x1000) & 1.82 & 3.30 & 0.0003 \\
\hline BALF & 27.2 & 46.9 & 0.05 \\
\hline \multicolumn{4}{|l|}{ PLF } \\
\hline Neutrophil Number & 64.1 & 27.9 & 0.183 \\
\hline BALF & 2.11 & 4.60 & 0.04 \\
\hline $\operatorname{PLF}\left(\times 10^{6}\right)$ & & & \\
\hline
\end{tabular}

\section{S99 A FUNCTIONAL VARIANT OF ELAFIN WITH IMPROVED ANTI-INFLAMMATORY ACTIVITY}

${ }^{1} \mathrm{Dm}$ Small, ${ }^{2} \mathrm{M}$ Zani, ${ }^{1} \mathrm{D}$ Quinn, 'S Weldon, 'Df McAuley, ${ }^{3} \mathrm{P}$ McNally, ${ }^{2} \mathrm{~T}$ Moreau, ${ }^{1} \mathrm{Cc}$ Taggart; ${ }^{1}$ Queen's University, Belfast, Northern Ireland; ' ${ }^{2}$ Francois-Rabelais University, Tours, France; ${ }^{3}$ Our Lady's Hospital for Sick Children, Dublin, Ireland

10.1136/thoraxjn-2013-204457.106

Introduction and Objectives Elafin is a well-known serine protease inhibitor produced by epithelial and inflammatory cells with anti-inflammatory properties. Previous work has shown that unregulated protease activity can cause the proteolytic cleavage of elafin, therefore impairing the innate immune function of the protein. Consequently, the aim of this study was to generate a variant of elafin that would demonstrate increased protease resistance whilst retaining many of the beneficial characteristics of the parent molecule.

Methods Two elafin variants (GG-elafin and QQ-elafin) were recombinantly synthesised in a yeast-based expression system and subsequently tested for antiprotease, transglutaminase and protease susceptibility. In addition, the LPS neutralisation activity of the GG-variant was evaluated in in vitro based assays and an in vivo mouse model of LPS-induced acute lung inflammation.

Results GG- and QQ-elafin retained similar antiprotease and transglutaminase activity compared to wild-type elafin (WT-elafin). When incubated with diseased bronchoalveolar lavage fluid (BALF), the elafin variants displayed significantly enhanced resistance to degradation when compared to WT-elafin. Intriguingly, both variants, but particularly GG-elafin, demonstrated improved LPS neutralisation by inhibiting cytokine expression in monocytic cells. Moreover, the GG-elafin showed improved anti-inflammatory properties in a mouse model of LPS-induced acute lung inflammation with significantly decreased inflammatory cell counts, namely neutrophils $(p=0.0362)$. Furthermore, total BAL protein levels were significantly decreased $(p=0.0336)$ and a reduction in pro-inflammatory cytokine/chemokine levels was observed in mice treated with GG-elafin compared to those treated with WT-elafin.

Conclusions Site-specific mutants of elafin, particularly GG-elafin, showed increased functionality when compared to WT-elafin and may be of future therapeutic relevance in the treatment of lung diseases, particularly acute lung injury (ALI).

\section{Mechanisms of cystic fibrosis}

\section{S100 NOVEL T HELPER CELL RESPONSES AGAINST PSEUDOMONAS AERUGINOSA IN HEALTHY INDIVIDUALS AND PATIENTS WITH CYSTIC FIBROSIS}

${ }^{1} \mathrm{HK}$ Bayes, ${ }^{2} \mathrm{M}$ Brodlie, ${ }^{2} \mathrm{C}$ Ward, ${ }^{2} \mathrm{PA}$ Corris, ${ }^{3} \mathrm{G}$ MacGregor, ${ }^{3}$ S Bicknell, ${ }^{1} \mathrm{~T}$ Evans; ${ }^{1}$ Institute of Infection, Immunology \& Inflammation, University of Glasgow, Glasgow, UK; ${ }^{2}$ Applied Immunobiology and Transplantation Group, Institute of Cellular Medicine, Newcastle University, Newcastle, UK; ${ }^{3}$ West of Scotland Cystic Fibrosis Centre, Gartnavel General Hospital, Glasgow, UK

\subsection{6/thoraxjnl-2013-204457.107}

Introduction and Objectives Pseudomonas aeruginosa (PA) colonisation is a hallmark of cystic fibrosis (CF) resulting in damaging neutrophilic inflammation. Patients with CF produce antipseudomonal antibodies but the role of $\mathrm{CD}^{+} \mathrm{T}$ cell responses to PA remains unclear. Novel $\mathrm{T}$ helper cell subsets, Th17 and Th22 cells, have important roles in host defense but may also enhance tissue damage. We aimed to define the antigen-specific memory $\mathrm{T}$ helper cell responses to Pseudomonas aeruginosa in healthy humans and patients with cystic fibrosis.

Methods $\mathrm{CD}_{14}{ }^{+}$monocytes and memory $\mathrm{CD} 4{ }^{+} \mathrm{CD}_{5} \mathrm{RO}^{+} \mathrm{T}$ cells were isolated from peripheral blood of CF patients with PA colonisation $(n=8)$ and healthy controls $(n=10)$. Monocytederived dendritic cells (DCs) were stimulated with live Pseudomonas strain PA103 and PA isolates derived from CF patients. Autologous T cells were co-cultured with activated DCs. The resultant $\mathrm{T}$ helper response was determined by measuring proliferation, immunoassay of cytokine output, and immunostaining of intracellular cytokines. Lavage samples from explanted CF lungs were assayed for IL-22 secretion. 